\title{
Implementation and utilization of genetic testing in personalized medicine
}

This article was published in the following Dove Press journal:

Pharmacogenomics and Personalized Medicine

13 August 2014

Number of times this article has been viewed

\author{
Noura S Abul-Husn',* \\ Aniwaa Owusu Obeng ${ }^{2,3, *}$ \\ Saskia C Sanderson' \\ Omri Gottesman² \\ Stuart A Scott ${ }^{1}$ \\ 'Department of Genetics and \\ Genomic Sciences, ${ }^{2}$ The Charles \\ Bronfman Institute for Personalized \\ Medicine, Icahn School of Medicine \\ at Mount Sinai, ${ }^{3}$ Department of \\ Pharmacy, Mount Sinai Hospital, New \\ York, NY, USA \\ *These authors contributed equally \\ to this manuscript
}

\begin{abstract}
Clinical genetic testing began over 30 years ago with the availability of mutation detection for sickle cell disease diagnosis. Since then, the field has dramatically transformed to include gene sequencing, high-throughput targeted genotyping, prenatal mutation detection, preimplantation genetic diagnosis, population-based carrier screening, and now genome-wide analyses using microarrays and next-generation sequencing. Despite these significant advances in molecular technologies and testing capabilities, clinical genetics laboratories historically have been centered on mutation detection for Mendelian disorders. However, the ongoing identification of deoxyribonucleic acid (DNA) sequence variants associated with common diseases prompted the availability of testing for personal disease risk estimation, and created commercial opportunities for direct-to-consumer genetic testing companies that assay these variants. This germline genetic risk, in conjunction with other clinical, family, and demographic variables, are the key components of the personalized medicine paradigm, which aims to apply personal genomic and other relevant data into a patient's clinical assessment to more precisely guide medical management. However, genetic testing for disease risk estimation is an ongoing topic of debate, largely due to inconsistencies in the results, concerns over clinical validity and utility, and the variable mode of delivery when returning genetic results to patients in the absence of traditional counseling. A related class of genetic testing with analogous issues of clinical utility and acceptance is pharmacogenetic testing, which interrogates sequence variants implicated in interindividual drug response variability. Although clinical pharmacogenetic testing has not previously been widely adopted, advances in rapid turnaround time genetic testing technology and the recent implementation of preemptive genotyping programs at selected medical centers suggest that personalized medicine through pharmacogenetics is now a reality. This review aims to summarize the current state of implementing genetic testing for personalized medicine, with an emphasis on clinical pharmacogenetic testing.
\end{abstract}

Keywords: personalized medicine, pharmacogenetics, pharmacogenomics, direct-to-consumer genetic testing, point-of-care genetic testing, preemptive genetic testing, implementation

\section{Introduction}

Clinical genetic testing historically has been limited to germline mutation detection for Mendelian diseases; however, candidate gene and genome-wide association studies (GWAS) have identified polymorphic deoxyribonucleic acid (DNA) sequence variants that significantly contribute to common disease susceptibility, other complex traits, and a number of drug response phenotypes. This has created the potential for implementing genetic testing to estimate personalized disease risk and/or to help guide individual pharmacotherapy. However, despite initial enthusiasm for common disease variant testing, ongoing concerns over inconsistent results, lack of clinical validity,
Correspondence: Stuart A Scott of Genetics and Genomic Sciences, Icahn School of Medicine at Mount Sinai, I Gustave L Levy Place, Box 1497, New York, NY, I0029, USA

Tel +I 2I 224 I 3780

$\mathrm{Fax}+\mathrm{I} 2122410139$

Email stuart.scott@mssm.edu 
and return of results without counseling suggest that genetic testing for common disease risk estimation currently is not ready for widespread adoption.

Pharmacogenetics is the study of the genetic determinants of drug response variability, and increasing enthusiasm for implementing clinical pharmacogenetic testing is evidenced by the personalized medicine programs that are now preemptively genotyping germline pharmacogenetic variants ${ }^{1}$ and the recent availability of clinical practice guidelines when pharmacogenetic test results are available. ${ }^{2}$ Other developments supporting the implementation of pharmacogenetic testing include the recent availability of rapid sample-to-answer genotyping platforms that could potentially be used at the point-of-care ${ }^{3}$ and the increasing use of clinical decision support (CDS) for health care providers deployed through electronic health records (EHRs). ${ }^{4}$ This review aims to summarize the current state of the personalized medicine genetic testing field, with an emphasis on pharmacogenetic testing and clinical implementation.

\section{Genetic testing: validity and utility}

In the US, genetic tests are commonly evaluated in Clinical Laboratory Improvement Amendments of 1988 (CLIA)certified laboratories by the Analytic validity, Clinical validity, Clinical utility, and associated Ethical, legal, and social implications (ACCE framework). ${ }^{5}$ Analytical validity is the ability to measure the genotype of interest accurately and reliably, which is generally high for assays interrogating germline pharmacogenetic variants. ${ }^{6,7}$ Clinical validity is the ability to detect or predict a phenotype associated with the genotype. Although it is a difficult metric to quantify for disease risk and pharmacogenetic testing, clinical utility is a measure of usefulness in the clinic and resulting changes in health outcomes.

The highest level of evidence to support clinical utility is derived from a prospective randomized controlled trial, yet these are often difficult to perform for pharmacogenetic hypotheses due to the challenges with achieving adequate power for low frequency adverse events and the potential ethical concerns with exposing individuals carrying at-risk genotypes.

Consequently, evidence to support clinical implementation of pharmacogenetic testing frequently has to be derived from alternative sources and study designs. Despite these challenges, the successful clinical translation of pharmacogenetics has been reported for $H L A-B * 5701$ screening to reduce the potentially lifethreatening hypersensitivity syndrome that occurs in $\sim 5 \%$ of the Caucasian human immunodeficiency virus patients treated with the antiretroviral agent abacavir. ${ }^{8}$ Moreover, the incorporation of pharmacogenomics into early-phase drug development and clinical trial design has been proposed to facilitate variant discovery, clinical translation, and to help inform later-phase studies and potential clinical implementation. ${ }^{9}$

\section{Personalized medicine: past and present}

Although personalized medicine has only recently been acknowledged in medical practice, physicians and researchers have observed interindividual differences in clinical traits and therapy responses for centuries. Historical examples include the four humors theory used by Hippocrates to diagnose and to prescribe therapy for individual patients; AL Fox's observation of interindividual variability in phenylthiocarbamide taste perception; R Bonicke's, W Reif's, and HB Hughes's description of interpatient differences in unchanged isoniazid urinary excretion that was later found to be due to differences in metabolic acetylation activity; and the increased episodes of primaquine-induced acute hemolytic crises witnessed in African American soldiers compared to Caucasians during World War II. ${ }^{10}$ Despite these and other more contemporary examples of interpatient variability in clinical course and therapy response, medicine of today is still largely based on the one-size-fits-all model where patients diagnosed with the same condition often are prescribed the same medication at the same dose. Although therapeutic successes have occurred with this model, for some medications this approach potentially can lead to preventable adverse drug reactions, reduced efficacy, noncompliance due to intolerance, and increased health care costs. For these reasons, a more patient-centric or personalized approach of medical practice has been proposed and is frequently debated. ${ }^{11}$

The completion of the Human Genome Project and the recent advances in genome sequencing technology have fueled translational research in genomics and the ongoing anticipation of medical practice that incorporates personal genomic data. Although understanding the genetic contribution to human disease is far from complete, polymorphic DNA sequence variants have been associated with common disorders and other complex traits by GWAS. ${ }^{12}$ In addition to advancing our scientific understanding of disease mechanisms and providing starting points for the development of medical treatments, the identification of certain susceptibility variants with significant disease associations also allows for the estimation of personal disease risks. As such, the 
personalized medicine paradigm now includes the utilization of individual genetic data in conjunction with other clinical, family, and demographic variables to inform decisions on disease prevention, diagnosis, treatment, and prognosis.

As noted in the "Clinical pharmacogenetic implementation programs" section, personalized medicine-based initiatives increasingly are being deployed by academic medical centers and other organizations. One resource for the personalized medicine community has been the Personalized Medicine Coalition, which was launched in 2004 in an effort to help advance the field. ${ }^{13}$

\section{Common disease risk variants Discovery of disease risk variants}

The first reported GWAS identified a significant association between the complement factor $\mathrm{H}(\mathrm{CFH})$ gene and agerelated macular degeneration (AMD) with an inspiring odds ratio of 4.6. ${ }^{14}$ With more than 25 risk variants now identified, AMD remains one of the most genetically wellcharacterized complex disorders. Two of the most notable variants that contribute substantially to AMD risk, $C F H$ and age-related maculopathy sensitivity $2(A R M S 2)^{15}$ have shed light on disease pathogenesis and may have implications in treatment response. ${ }^{16}$

Type 1 diabetes (T1D) is another example of a prevalent complex disease for which genetic susceptibility has been the subject of intensive study. Human leukocyte antigen (HLA) associations with T1D were first observed in the $1970 \mathrm{~s},{ }^{17}$ and high-risk $H L A$ class II variants remain the strongest genetic association with T1D, accounting for $30 \%-50 \%$ of the genetic risk and conferring up to a 20 -fold increased risk for T1D. ${ }^{18}$ Subsequent candidate-gene studies and GWAS have revealed more than 40 non- $H L A$ risk variants for T1D, ${ }^{19,20}$ which jointly confer only limited additional risk beyond the $H L A$ variants, but nevertheless have provided important insights into the natural history and pathophysiology of the disease. ${ }^{21}$ The most significant of these are variants in the insulin $(I N S)$ and protein tyrosine phosphatase nonreceptor type 22 (PTPN22) genes, with PTPN22 variants also being independently associated with other autoimmune diseases. ${ }^{22}$

There are many other examples of common diseases for which GWAS have identified disease-variant associations; ${ }^{12}$ however, the majority of these associations are of modest effect sizes, with a median odds ratio of only 1.33 - and few being $>3 .{ }^{23}$ Moreover, most identified disease risk variants can only explain a small proportion of the estimated genetic heritability of common diseases. ${ }^{24}$ In addition, some common conditions, such as psychiatric diseases, have been more challenging to investigate by GWAS, requiring much larger sample sizes to identify significant associations. Despite the small amount of heritability explained by most reported common disease risk variants and the ongoing difficulties with risk prediction, these studies have illuminated new biology and previously unknown disease pathways. $^{25}$

\section{Genetic risk prediction}

Traditional disease risk assessments incorporate environmental and clinical factors known to be associated with common diseases and are intended to help clinicians more accurately assess patient risk for the purpose of prevention, diagnosis, treatment, and prognosis. A widely used example of this is the Framingham risk score, which is a sex-specific algorithm that estimates 10 -year cardiovascular disease risk. ${ }^{26}$ While the importance of genetics in disease risk prediction has long been recognized by the presence or absence of a family history, genetic variables have only recently been incorporated into risk prediction models.

Genetic risk prediction began, with some success, using common variants of large effect size that were mostly discovered in the pre-GWAS era, such as the $H L A$ effect in T1D. ${ }^{21}$ To date, the most well-known genetic variables utilized in disease risk prediction are $B R C A 1$ and $B R C A 2$, which were originally identified by linkage studies ${ }^{27,28}$ to be associated with hereditary breast and ovarian cancer syndrome. ${ }^{29}$ Genetic risk prediction models for other common diseases are beginning to surface, using various subsets of risk variants, sometimes in combination with clinical and environmental factors. In the case of AMD, a recent study showed a significant increase in disease predictability by incorporating six risk variants to an algorithm previously based on clinical and environmental factors. ${ }^{30}$

\section{Direct-to-consumer genetic testing}

Clinical genetic testing traditionally has been available through physicians and genetic counselors; however, the landscape of genetic testing has changed considerably with the emergence of direct-to-consumer (DTC) genetic testing. With DTC testing, consumers can order genetic tests directly, and results often are returned without health care provider involvement or genetic counseling. The modern era of DTC genetic testing began in 2007 with the launch of deCODE genetics, Navigenics, and 23andMe. By 2011, there were at least 27 reported companies offering DTC genetic testing 
to US consumers. ${ }^{31}$ Even though some of these companies are no longer in existence or offering DTC products, a broad range of DTC genetic tests remain available today.

An evolving aspect of DTC genetic testing has been the use of genetic risk prediction tools for a wide array of common diseases. Unfortunately, DTC genetic risk estimates typically have low-to-moderate predictive ability for common diseases, due to the small effect sizes of most disease risk variants. In addition, the reported effect sizes of these variants are dependent on the nature of the studied population, including ancestry. As such, an association detected in one population will not necessarily be transferable to another racial or ethnic group. Furthermore, for any given disease, there has been little concordance between DTC companies in their variant selection, effect sizes, average population risk, and algorithms used to calculate disease risk, which has resulted in substantial differences in individual risk prediction between the DTC companies. ${ }^{32,33}$

DTC pharmacogenetic testing is available for a variety of drug-gene pairs - including those with and without wellestablished guidelines. ${ }^{34}$ Similar to the DTC disease risk estimates, DTC pharmacogenetic tests can differ in the genes and variants interrogated, which affects the interpretation of results and accuracy of predictions and is important when considering variants with allele frequencies that vary between racial and ethnic groups. ${ }^{34}$

\section{Pharmacogenetic variants}

The field of pharmacogenetics is generally believed to have originated in the late 1950s when the term "pharmacogenetics" was first published by F Vogel. ${ }^{35}$ Many pharmacogenetic discoveries followed, including the 1977 identification of polymorphic debrisoquine hydroxylation inherited as an autosomal recessive trait. ${ }^{36}$ The responsible enzyme was later identified as cytochrome P450-2D6 (CYP2D6) and is now believed to be involved in the metabolism of $\sim 25 \%$ of all commonly used drugs. ${ }^{37}$ Numerous CYP2D6 genetic studies have been reported with more than 100 variant star (*) alleles now cataloged by the Human Cytochrome P450 (CYP) Allele Nomenclature Committee. ${ }^{38}$

In an effort to potentially utilize $C Y P 2 D 6$ status to guide pharmacotherapy, some CLIA-certified genetic testing laboratories offer $C Y P 2 D 6$ genotyping with an interpretation that categorizes patients into one of four metabolizer phenotypes: ultrarapid; extensive; intermediate; or poor. ${ }^{37}$ Like other pharmacogenetic examples, the application of CYP2D6 genotype results is dependent on the clinical context and specific medication for a given patient. Some known pharmacogenetic variants are associated with drug efficacy, while others are implicated in toxicity and/or adverse drug reactions.

There are more than 50 other known human cytochrome P450 enzymes and variant alleles in these genes are being discovered continuously in various populations. Two notable CYP2C subfamily enzymes with polymorphic genes that often are included in clinical pharmacogenetic genotyping panels are CYP2C $9{ }^{39}$ and CYP2C $19 .{ }^{40} \mathrm{CYP} 2 \mathrm{C} 9$ metabolizes many clinically relevant drugs including phenytoin, warfarin, tolbutamide, and losartan. Among other associations, $C Y P 2 C 9$ reduced-function alleles have been implicated in interindividual warfarin dosing variability and bleeding risk. ${ }^{41}$ The vitamin $\mathrm{K}$ epoxide reductase complex 1 (VKORC1) ${ }^{42}$ promoter variant (c.-1639G $>\mathrm{A}$ ) is also strongly predictive of therapeutic warfarin dosing requirements ${ }^{43}$ and variants from these two genes have been incorporated with other clinical variables into warfarin dosing algorithms. ${ }^{44}$ Importantly, retrospective studies have shown that pharmacogenetic-guided warfarin dosing is more accurate than both fixed dosing and the dosing table in the warfarin label. ${ }^{45}$ However, recent randomized clinical trials indicate that although pharmacogenetic-guided dosing is more accurate than typical fixed dosing, ${ }^{46}$ an algorithm with only three $C Y P 2 C 9$ and $V K O R C 1$ variants common among Caucasians is not superior to a clinical variable dosing algorithm, particularly among individuals of African ancestry. ${ }^{47}$ As such, a major issue behind the pharmacogenetic-guided warfarin dosing continues to be the appropriate selection of $C Y P 2 C 9$ and $V K O R C 1$ variants for a given ethnicity and the ongoing debate over clinical utility. ${ }^{48}$ These important trials also suggest that more ethnic-specific dosing algorithms are needed.

The CYP2C19 enzyme contributes to the metabolism of a large number of clinically relevant drugs and drug classes such as antidepressants, benzodiazepines, mephenytoin, proton pump inhibitors, and the antiplatelet prodrug clopidogrel. Importantly, the common $C Y P 2 C 19 * 2$ allele has been associated with reduced active clopidogrel metabolites, higher ontreatment platelet aggregation, and adverse clinical outcomes compared to noncarriers among clopidogrel-treated patients with acute coronary syndromes and/or those undergoing percutaneous coronary intervention (PCI), ${ }^{49,50}$ and practice guidelines on $C Y P 2 C 19$-directed antiplatelet therapy are available (Table 1).

Other notable enzymes and transporters with known variants and potential clinical utility include dihydropyrimidine dehydrogenase (DPYD) and fluorouracil response, ${ }^{51}$ thiopurine S-methyltransferase (TPMT) and thiopurine toxicity, ${ }^{52} \mathrm{UDP}-$ 
glucuronosyltransferase (UGT1A1) and irinotecan toxicity, ${ }^{53}$ and the solute carrier organic anion transporter family member 1B1 (SLCO1B1) gene implicated in statin-induced myopathy. ${ }^{54,55}$ Other genes and variants implicated in drug response variability are continually being discovered, most commonly now by genome-wide approaches. ${ }^{56-58}$ With respect to drug efficacy, these include the identification of IL28B and interferon- $\alpha$ response for hepatitis $\mathrm{C}$ infection, ${ }^{59}$ SLCO1B1 and methotrexate response, ${ }^{60}$ GLCCI1 and glucocorticoid response, ${ }^{61}$ and $C Y P 4 F 2$ and its role in warfarin dosing. ${ }^{62,63}$

Examples involving drug toxicity or adverse drug reactions include $H L A-B * 5701$ and flucloxacillin-induced liver injury, ${ }^{64}$ and $H L A-A * 3101$ and carbamazepine-induced hypersensitivity. ${ }^{65}$ As noted previously, the frequencies of these variant alleles can significantly differ between racial and ethnic groups, which influence the variability in drug response observed between populations in addition to between individuals. ${ }^{66,67}$ Online resources dedicated to summarizing multi-ethnic pharmacogenetic allele frequencies are available (eg, FINDbase-PGx), ${ }^{68}$ and they are also summarized on the Pharmacogenomics Knowledge Base (PharmGKB) website (https://www.pharmgkb.org/).
Many of the genes and variants noted above currently are being considered for clinical implementation or are already implemented at several medical centers. However, despite this enthusiasm, routine clinician uptake of available pharmacogenetic testing has been low, likely due to a number of factors including concerns over clinical validity/utility and insufficient evidence, a lack of professional education and guidelines, and other logistic, regulatory, and reimbursement issues. ${ }^{69}$ To specifically address the educational needs for pharmacogenetic testing implementation, clinical practice guidelines that detail therapeutic options based on pharmacogenetic test results are being developed by a number of organizations (Table 1). Although some guidelines evaluate the evidence and determine whether testing is warranted or not, ${ }^{70,71}$ others simply provide evidence-based recommended clinical actions for when a patient's genotype is already known. ${ }^{2,72,73}$ Examples include the Royal Dutch Association for the Advancement of Pharmacy-Pharmacogenetics Working Group (KNMP-PWG) guidelines that report on 53 drugs and eleven genes ${ }^{72}$ and the evidence-based gene/ drug guidelines reported by the Clinical Pharmacogenetics Implementation Consortium (CPIC) of the National Institutes of Health's Pharmacogenomics Research Network

Table I Candidate genes for pharmacogenetic implementation with available practice guidelines and recommendation statements

\begin{tabular}{|c|c|c|c|}
\hline Gene(s) & Drug & Organization & $\begin{array}{l}\text { Practice guidelines/ } \\
\text { recommendation statements }\end{array}$ \\
\hline CFTR & Ivacaftor & CPIC & Clancy et al; $2014^{118}$ \\
\hline \multirow[t]{2}{*}{ CYP2C9/VKORCI } & Warfarin & ACMG & Flockhart et al; $2008^{119}$ \\
\hline & & CPIC & Johnson et al; $201 \mathrm{I}^{120}$ \\
\hline \multirow[t]{3}{*}{ CYP2CI9 } & Clopidogrel & CPIC & Scott et al; $201 \mathrm{I},,^{121} 2013^{122}$ \\
\hline & & ACCF/AHA & Holmes et al; $2010^{123}$ \\
\hline & TCAs & CPIC & Hicks et al; $2013^{124}$ \\
\hline \multirow[t]{5}{*}{ CYP2D6 } & Codeine & CPIC & Crews et al; $2012^{125}$ \\
\hline & SSRIs & CPIC & In preparation; 2014a \\
\hline & & EGAPP & EGAPP Working Group; $2007^{70}$ \\
\hline & Tamoxifen & ACMG & Lyon et al; $2012^{126}$ \\
\hline & TCAs & CPIC & Hicks et al; $2013^{124}$ \\
\hline CYP3A & Tacrolimus & CPIC & In preparation; $2014^{a}$ \\
\hline DPYD & Fluoropyrimidine & CPIC & Caudle et al; $2013^{127}$ \\
\hline \multirow[t]{4}{*}{ HLA-B } & Abacavir & CPIC & Martin et al; $2012^{128}$ \\
\hline & Allopurinal & CPIC & Hershfield et al; $2013^{129}$ \\
\hline & Carbamazepine & CPIC & Leckband et al; $2013^{130}$ \\
\hline & Phenytoin & CPIC & In preparation; $2014^{\mathrm{a}}$ \\
\hline IFNL3 (IL28B) & Interferon- $\alpha$ & CPIC & Muir et al; $2014^{|3|}$ \\
\hline SLCOIBI & Simvastatin & CPIC & Wilke et al; $2012^{132}$ \\
\hline TPMT & Azathioprine/6-mercaptopurine & CPIC & Relling et al; 20II, ${ }^{133} 2013^{134}$ \\
\hline UGTIAI & Irinotecan & EGAPP & EGAPP Working Group; $2009^{71}$ \\
\hline Multiple (eleven genes) & Multiple (53 drugs) & KNMP-PWG & Swen et al; $201 \mathrm{I}^{72}$ \\
\hline
\end{tabular}

Note: ${ }^{B}$ Based on information available from Pharmacogenomics Knowledge Base website (http://www.pharmgkb.org/cpic/pairs), and K Caudle, CPIC Coordinator, personal communication.

Abbreviations: TCA, tricyclic antidepressants; SSRI, selective serotonin reuptake inhibitors; ACCF, American College of Cardiology Foundation; ACMG, American College of Medical Genetics and Genomics; AHA, American Heart Association; CPIC, Clinical Pharmacogenetics Implementation Consortium; EGAPP, Evaluation of Genomic Applications in Practice and Prevention; KNMP-PWG, Royal Dutch Association for the Advancement of Pharmacy-Pharmacogenetics Working Group. 
(PGRN) (Table 1). ${ }^{2}$ In addition, evidence-based practice recommendation statements for pharmacogenetic testing have also been published by the Evaluation of Genomic Applications in Practice and Prevention (EGAPP) Working Group, ${ }^{74}$ launched by the Centers for Disease Control and Prevention Office of Public Health Genomics (Table 1).

\section{Clinical pharmacogenetic testing and implementation \\ Clinical genetic testing regulation}

In the US, CLIA regulations include federal standards applicable to all laboratories that test human specimens for health assessment or to diagnose, prevent, or treat disease. The CLIA program supports clinical laboratory quality by developing technical standards and laboratory practice guidelines, conducting laboratory quality improvement studies, monitoring proficiency testing practices, among other initiatives and resources. CLIA-certified laboratories can offer genetic tests using either validated laboratory-developed tests or commercially available products approved by the US Food and Drug Administration (FDA).

Table 2 summarizes the commercially available DNAbased pharmacogenetic tests that currently are US FDA-approved for in vitro diagnostic testing. Clinical laboratories can also participate in the pharmacogenetic proficiency testing programs offered by the College of American Pathologists, which provides graded and educational surveys for $C Y P 2 C 9$ and VKORC1, CYP2C19, CYP2D6, and UGT1A1. ${ }^{75}$ To address the need for quality control reference materials, the Coriell Cell Repositories and the Genetic Testing Reference Materials Coordination Program of the Centers for Disease Control and Prevention have characterized a panel of commercially available cell lines for genes and variants commonly interrogated by pharmacogenetic assays. ${ }^{76}$ An expanded Genetic Testing Reference Materials Coordination Program pharmacogenomics reference material project is currently ongoing that is interrogating a much larger panel of genes and variants, using multiplexed targeted assays, genotyping microarrays, and next-generation sequencing gene panels.

\section{Point-of-care genetic testing}

A common challenge when implementing pharmacogenetic testing in a traditional health care environment is the frequent need for rapid return of results. In an effort to address this, biotechnology companies have been developing genotyping platforms that offer rapid sample-to-result assays for possible use at the point-of-care (POC). ${ }^{6,77,78}$ For example, the reassessment of anti-platelet therapy using an individualized strategy based on genetic evaluation (RAPID GENE) trial successfully implemented POC CYP 2 C $19 * 2$ genetic testing for cardiac patients initiating clopidogrel therapy following PCI. ${ }^{3}$ Patients were randomized to either CYP2C19*2 genotyping using a rapid $P O C$ cheek swab device or standard treatment with clopidogrel. Identified CYP2C19*2 carriers in the genotyping arm were treated with prasugrel while

Table 2 Pharmacogenetic tests approved by the US FDA for IVD use ${ }^{a}$

\begin{tabular}{|c|c|c|c|c|}
\hline Gene(s) & Assay & Alleles interrogated & Company & Date approved \\
\hline CYP2C9 and & Verigene ${ }^{\circledR}$ Warfarin & CYP2C9*2, *3, VKORCI & Nanosphere, Inc. & September 2007 \\
\hline \multirow[t]{7}{*}{ VKORCI } & Metabolism Test & c. $174-136 C>T(\mid 173 C>T)$ & & \\
\hline & INFINITI ${ }^{\otimes}$ Warfarin Assay & CYP2C9*2, *3, VKORCI & AutoGenomics, Inc. & January 2008 \\
\hline & & c. $-1639 G>A$ & & \\
\hline & eSensor ${ }^{\circledR}$ Warfarin & CYP2C9*2, *3, VKORCI & GenMark Diagnostics, Inc. & July 2008; \\
\hline & Sensitivity Test & c. $-1639 G>A$ & & December 201I \\
\hline & eQ-PCR TM LC Warfarin & CYP2C9*2, *3, VKORCI & TrimGen Corporation & February 2009 \\
\hline & Genotyping Kit & c. $-1639 \mathrm{G}>\mathrm{A}$ & & \\
\hline \multirow[t]{5}{*}{ CYP2CI9 } & AmpliChip ${ }^{\circledR}$ CYP450 Test & $* 2, * 3$ & Hoffmann-La Roche Ltd & January 2005 \\
\hline & INFINITI ${ }^{\circledR}$ CYP2CI9 Assay & $* 2, * 3, * 17$ & AutoGenomics, Inc. & October 2010 \\
\hline & Verigene ${ }^{\circledR}$ CYP2C19 Test & $* 2, * 3, * 17$ & Nanosphere, Inc. & November 2012 \\
\hline & Spartan RX CYP2C19 Assay & $* 2, * 3, * 17$ & Spartan Bioscience Inc. & August 2013 \\
\hline & xTAG ${ }^{\circledR}$ CYP2C19 Kit v3 & $* 2, * 3, * 17$ & Luminex Molecular Diagnostics, Inc. & September 2013 \\
\hline \multirow[t]{2}{*}{ CYP2D6 } & AmpliChip $^{\circledR}$ CYP450 Test $^{\mathrm{b}}$ & $\begin{array}{l}* 2-* 11, * 15, * 17, * 19, * 20, \\
* 29, * 35, * 36, * 40, * 41, \text { duplication }\end{array}$ & Hoffmann-La Roche Ltd & January 2005 \\
\hline & xTAG ${ }^{\circledR}$ CYP2D6 Kit v3 & $\begin{array}{l}* 2-* 11, * 15, * 17, * 29, * 35 \\
* 41, \text { duplication }\end{array}$ & Luminex Molecular Diagnostics, Inc. & May 2013 \\
\hline
\end{tabular}

Notes: aAs listed on the US FDA IVD Product Database: http://www.fda.gov/MedicalDevices/ProductsandMedicalProcedures/InVitroDiagnostics/default.htm; bthe CYP2D6 star $\left(^{*}\right)$ allele nomenclature for the AmpliChip ${ }^{\circledR}$ is based on the available nomenclature at the time of product release. The $* 4 I$ allele reported by the AmpliChip ${ }^{\circledR}$ is not consistent with the current $* 4$ I haplotype nomenclature as it does not interrogate the $2988 \mathrm{G}>\mathrm{A}$ variant that was discovered after the development of the Amplichip ${ }^{\circledR} .{ }^{135}$ Abbreviations: FDA, Food and Drug Administration; IVD, in vitro diagnostic. 
noncarriers were treated with clopidogrel. As a proof of concept trial, no carriers in the genotyping arm had high on-treatment platelet reactivity at day 7 compared with $30 \%$ of patients undergoing standard treatment. ${ }^{3}$

The success of RAPID GENE suggests that POC genetic testing can be performed effectively by nursing staff and that personalized antiplatelet therapy can reduce high on-treatment platelet reactivity in this patient population; however, this trial was not designed to test whether this strategy results in better clinical outcomes. A number of trials are underway to address this important question, including: the reassessment of antiplatelet therapy using an individualized strategy in patients with ST-segment elevation myocardial infarction (RAPID STEMI; ClinicalTrials.gov identifier, NCT01452139); the tailored antiplatelet therapy following PCI (TAILOR-PCI; NCT01742117), and the customized choice of P2Y12 oral receptor blocker based on phenotype assessment via pointof-care testing (PRU-MATRIX; NCT01477775).

Despite the support for POC genetic testing by the RAPID GENE trial, logistical and regulatory issues need to be considered when implementing POC genetic testing into actual routine clinical care. Although the genotyping platform used in RAPID GENE has recently been approved by the US FDA (Table 2), it currently is not supported as a true POC device since it does not qualify for a CLIA waiver. Furthermore, because any genetic testing is broadly categorized by CLIA as high complexity testing, all pharmacogenetic POC testing platforms will likely not be amenable to CLIA waivers. As such, a CLIA-certified genetics laboratory will be required to oversee POC testing by managing performance, interpretation, quality control/assurance, and proficiency, which ultimately may diminish the inherent advantages of a POC genetic testing device. Nevertheless, the dramatic reduction in turnaround times of these platforms is undoubtedly a major benefit when implementing pharmacogenetic testing for selected clinical scenarios.

Another challenge for POC pharmacogenetic testing is the content of the assays. Some of the most robust pharmacogenetic associations with large effect sizes involve the aforementioned $H L A$ alleles and flucloxacillin-induced liver injury/abacavir hypersensitivity $(H L A-B * 5701)^{79}$ and carbamazepine hypersensitivity (HLA-B*1502/HLA$\left.A^{*} 3101\right) .{ }^{65,80}$ Unfortunately, HLA genotyping is beyond the technical capacity of current POC platforms due to the extreme polymorphic nature of the HLA loci (including copy number variation) and the necessary requirement for highly multiplexed genotyping and/or sequencing. Additionally, other notable gene/drug examples will require the interrogation of multiple variant alleles for clinical validity in patient populations with diverse ancestries. As such, future POC genetic testing platforms ideally will need to be capable of multiplexed genotyping for alleles with clear clinical actionability and those that are prevalent in multiethnic patient populations.

\section{Preemptive genetic testing}

Another testing strategy that can circumvent some of the issues with both traditional external laboratory and POC pharmacogenetic testing is preemptive genotyping. Although this model also has inherent challenges for effective clinical implementation, preemptive pharmacogenetic testing recently has been deployed at selected academic medical centers (see the "Clinical pharmacogenetic implementation programs" section). This approach preemptively deposits genotype data into EHRs through prospective or biobank patient sampling and CLIA-certified genetic testing, and alerts prescribers at the POC through sophisticated electronic CDS when a drug is ordered for a patient with an at-risk genotype. The immediate knowledge of a pharmacogenetic interaction coupled with possible therapeutic options without significant disruption of routine clinical care is the clear advantage to this strategy. However, the necessary investments in infrastructure, informatics, health care provider participation and education, and preemptive testing in a CLIA-certified environment suggest that this mode of clinical pharmacogenetic delivery will be limited to large academic medical centers for the foreseeable future.

\section{Information technology and clinical decision support}

Genetic information is complex in nature, different to the other types of patient data that health care providers normally consider, and its significance to the individual patient changes frequently - all factors that today act as significant barriers to the widespread use of genetic information in clinical care.$^{81}$ In addition, the concept of genetic information having clinical utility outside of the clinical genetics domain is new and, as such, most practicing providers have no formal education in its use. ${ }^{82}$

Health care information technology offers a potential solution to these barriers. Almost in parallel with the rise of genomic data, US health care has seen a surge in digitized clinical data through the incentivization and subsequent widespread implementation of EHRs. As noted previously, one of the quality improvement mechanisms offered by EHRs is the potential for POC clinical decision support (CDS). 
The CDS provides clinicians with knowledge presented at appropriate times, typically through computerized alerts and reminders. ${ }^{83}$ To date, much of this has centered on drug-drug or drug-allergy interactions; however, with the emergence of disease-risk and drug response-associated genetic variants, the CDS has the potential to consolidate and translate genomic knowledge and integrate this knowledge into existing clinical workflows that will allow clinicians to make genomeinformed decisions at the POC. Importantly, this will remove the significant burden from providers of remaining current in a rapidly changing field, where the potential permutations of clinical decision rules far exceed what could be expected of clinical providers to remember.

\section{Clinical pharmacogenetic implementation programs}

To identify and address barriers to implementing pharmacogenetics, the PGRN recently established the Translational Pharmacogenomics Program comprised of six diverse academic medical centers. ${ }^{1}$ For this initiative, each implementation step is being systematically evaluated to develop a practical evidence-based toolbox of best practices for implementing pharmacogenetics across a variety of health care systems. Other instruments that will be developed for dissemination through publication include a best practices manual for clinical pharmacogenetic testing, and questionnaires and surveys to assess implementation metrics and effectiveness.

One of the first structured pharmacogenetics programs was a pharmacist-driven clinical service at St Jude Children's Research Hospital that initially offered TPMT and UGT1A1 genotyping. ${ }^{84}$ The expanded St Jude PG4KDS program is now migrating CYP2C19, CYP2D6, SLCO1B1, and TPMT genotype data from a microarray-based panel ${ }^{85}$ into the EHR as prospective patients are enrolled, and deploying CDS when an intersection between a prescription and an at-risk genotype occurs. ${ }^{86}$ The Pharmacogenomic Resource for Enhanced Decisions in Care and Treatment (PREDICT) program at the Vanderbilt University Medical Center is preemptively genotyping CYP2C19 in $~ 3,000$ patients scheduled for cardiac catheterization, depositing data into the EHR, and providing POC CDS when clopidogrel is prescribed for patients with variant $C Y P 2 C 19$ genotypes. ${ }^{87}$ PREDICT is currently being expanded to include warfarin/CYP2C9-VKORC1 and simvastatin/SLCO1B1 drug/ gene pairs. Similarly, the University of Florida and Shands Hospital's Personalized Medicine Program also is centered on clopidogrel and CYP2C19 for a pharmacogenetics pilot program; ${ }^{88}$ however, their multiplexed genotyping panel also includes a much larger number of genes and variants tested under research consent for storage in the EHR and possible use in future implementation efforts. ${ }^{89}$ The 1,200 Patients Project at the University of Chicago is genotyping a large panel of germline pharmacogenetic variants among patients receiving outpatient medical care who are taking prespecified prescription medications. ${ }^{90}$ Patient-specific results are then made available to the enrolling provider through an online genomic prescribing system. ${ }^{91}$

The Clinical Implementation of Personalized Medithrough Electronic Health Records and Genomics (CLIPMERGE)Pharmacogenomics program at the Icahn School of Medicine at Mount Sinai is preemptively genotyping a panel of germline pharmacogenetic variants, storing data in an external datamanagement platform that interfaces with the EHR, and delivering CDS at the POC through the EHR. However, unlike some of the other implementation programs, patient recruitment is accomplished through the Mount Sinai's BioMe Biobank Program, which currently includes $\sim 30,000$ multi-ethnic patients. ${ }^{92}$ Patients for the pilot program are selected based on their regular attendance at Mount Sinai for their primary care and who are currently taking (or likely to take) clopidogrel, warfarin, simvastatin, tricyclic antidepressants, and/or selective serotonin reuptake inhibitors. As part of the CLIPMERGE program, enrolled physicians have been extensively surveyed regarding their views on genomic medicine and CDS. ${ }^{93}$ These data have demonstrated that although physicians are generally familiar with and comfortable using CDS, there is a significant deficit in physician familiarity and comfort with interpreting and utilizing genomic information. ${ }^{93}$ These insights suggest that to achieve the promise of personalized medicine, education on genomics must be a priority for clinical providers at all stages of their careers, and mechanisms that efficiently integrate personalized medicine into POC workflows via CDS and the EHR must continue to be developed and refined.

The Coriell Personalized Medicine Collaborative $\left(\mathrm{CPMC}^{\odot}\right)^{94}$ is a multi-institutional observational research study evaluating the utility of personalized genomic risk information in health care..$^{95}$ To interpret and report pharmacogenetic variants to study participants, the $\mathrm{CPMC}^{\odot}$ has recently deployed the Pharmacogenomics Appraisal, Evidence Scoring and Interpretation System. ${ }^{96}$ Guided by the expertise of an advisory group and thorough assessment of available evidence, drugs, and genes are identified for potential clinical utility, and selected drug/gene pairs are provided to $\mathrm{CPMC}^{\odot}$ participants using risk reports containing genetic results, interpretation, educational summaries, detailed information 
on genetic and nongenetic risk factors affecting drug response, and frequencies of drug response phenotypes in the population most relevant to the participant.

\section{Personalized genetic testing results and patient behavior}

Common concerns when implementing genetic testing for personalized medicine are often centered on the infrastructure, logistics, and other related issues noted previously; however, another important component that requires consideration is the perspective of the patient when receiving genetic test results.

Although an overview of genetic counseling is beyond the scope of this review, there are three key psychological questions to consider when implementing personalized medicine genetic testing:

1. Are patients open to and interested in receiving personalized genetic testing information?

2. What is the effect of personalized genetic testing information on psychological well-being?

3. Does personalized genetic testing information motivate patients to improve their health-related behaviors?

All of these questions are of importance both because they speak to important ethical considerations, and because they speak to aspects of the potential utility of genetic testing information for improving health-related outcomes.

\section{Patient interest in receiving personal genetic results}

Available evidence suggests that patients are more interested in receiving personal genetic results when the disease risk information has sufficient clinical utility to inform future preventative and/or therapeutic interventions that might mitigate any potential increased genetic risk. ${ }^{97}$ Although the uptake of genetic testing for a given disease is influenced by multiple factors, patients appear to be influenced by the clinical validity of genetic tests as well as the severity of the diseases being tested. For example, while the uptake of the clinical genetic testing for hereditary colon cancer ranges from $\sim 50 \%-80 \%,{ }^{98,99}$ the uptake of clinical genetic testing for Huntington's disease, which is a late-onset dominant disorder without any preventative intervention, is only $\sim 15 \%-20 \%$. ${ }^{100,101}$ Similarly, patients are also generally less interested in receiving personal disease risk information on more severe neuropsychiatric conditions, such as Alzheimer's disease. ${ }^{102}$ However, the observation that a minority of patients do opt for genetic risk information even when clinical utility is low indicates that some people derive personal utility from this information by enabling more educated future planning. ${ }^{103}$

Despite the ongoing research on the impact of DTC genetic testing on health care systems and patient behavior, ${ }^{104-107}$ few studies have reported on patient interest in pharmacogenetic testing. However, recent data indicate that the general public generally has a high level of interest in receiving pharmacogenetic information, ${ }^{108}$ suggestive of positive public attitudes toward the implementation of pharmacogenetics in clinical care. Although it is likely that patients will be reasonably comfortable with personal pharmacogenetic testing, the extent to which patients will be involved in the decision to actually receive pharmacogenetic information versus physicians making these decisions on their behalf is currently unclear. This is particularly relevant in the context of the preemptive genotyping strategies detailed previously where CDS is directed to the physician in the absence of a clinical geneticist or counselor. Given the inherent distinctions between pharmacogenetic testing and Mendelian disease genetic testing, formal genetic counseling for pharmacogenetic results is likely not going to be routine practice, underscoring the importance of continued education in pharmacogenetics for primary care and other providers so they are equipped to communicate these types of results.

\section{Psychological outcomes}

In the clinical genetics arena, the psychological harms from genetic testing information are generally uncommon, ${ }^{97}$ which is likely due to the extensive genetic counseling received and through self-selection, where patients who feel they could not cope with adverse genetic test results are more likely to choose not to actually receive these results. ${ }^{109}$ Similarly, available evidence suggests that the DTC genetic tests for common diseases and complex traits do not cause significant psychological harm. ${ }^{105}$ Given the high benefit-to-risk ratio with pharmacogenetic testing, it is likely that there will also be minimal psychological harms from this mode of genetic testing; however, the lack of available data suggest that further study on psychological outcomes following pharmacogenetic testing is warranted, particularly as related to managing potential ancillary disease risk information. ${ }^{110}$

\section{Behavioral outcomes}

Studies on genetic testing for lung cancer risk suggest that personal genetic information does not independently have a significant impact on motivation to change behavior (eg, quit smoking). ${ }^{111,112}$ Similarly, DTC genetic testing has not been shown to motivate people to make significant lifestyle 
changes, ${ }^{104,105}$ however, it is possible that genetic information may motivate people under certain circumstances. Psychological models of health behavior suggest that genetic information may motivate people to engage in healthier behaviors if the genetic information pertains to conditions or diseases that people feel threatened by, and when there is something they can do to reduce the disease risk. For example, in a study on Alzheimer's disease, 29\% of APOE \&4 positive (higher risk) participants started to take dietary supplements in an effort to reduce their disease risk, compared to only $8 \%$ in the $A P O E$ $\varepsilon 4$ negative (lower risk) group. ${ }^{113}$ This increased genetic risk for Alzheimer's disease has also been associated with a higher likelihood of purchasing long-term care insurance. ${ }^{114}$

Although not well-studied, pharmacogenetic test results may have particular promise for influencing patient health behaviors. For example, recent data suggest that pharmacogenetic testing may improve medication adherence by increasing patient confidence in the efficacy of their prescribed medication and that their medication will work for them. ${ }^{115}$ If a patient believes that pharmacogenetic testing provides personally relevant information about a disease that is threatening to them, and that the test results might effectively lead to increased efficacy and/or reduced likelihood of adverse side-effects from a prescribed medication, then it is plausible that pharmacogenetic testing could have a positive impact on medication adherence.

\section{Conclusion and future direction}

Although many agree that the concept of personalized medicine is important and an essential field to be developed, there have been few examples where the incorporation of personal genetic information has led to robust improvements in clinical care. The identification of common disease risk variants has undoubtedly revealed new biology and potential new avenues for therapeutic intervention; however, the modest effect sizes of most identified variants has resulted in personalized disease risk calculations with questionable clinical validity and utility. As such, testing for these variants is not common among clinical genetics laboratories and has largely been restricted to DTC genetic testing companies. Ongoing genome sequencing studies likely will identify rare variants that may improve the predictability for some diseases, but it is clear that further development of risk prediction algorithms is needed. In addition to genomic sequence data, these algorithms could include other forms of clinical, demographic, epigenomic and environmental data, and could potentially utilize more sophisticated network-based modeling approaches. ${ }^{116,117}$
The enthusiasm for pharmacogenetic testing and its potential utility has prompted a number of recent efforts to facilitate clinical implementation, including clinical practice guidelines for pharmacogenetic results, rapid turnaround time genotyping platforms, and institutional programs supporting preemptive pharmacogenetic genotyping with result returning and CDS at the POC. Together, these initiatives indicate that personalized medicine finally may be materializing at selected institutions through pharmacogenetics. However, these exciting advances for the field should be balanced by the clear needs for continued discovery research for variants and other factors that significantly influence drug response variability, particularly in populations from different racial groups and ethnicities, enhanced pharmacogenetics education for health care providers at all levels of their careers, and further development of information technology and other related mechanisms that can facilitate efficient implementation of result returning without interruption of routine clinical care. These exciting steps forward for personalized medicine are hopefully just the beginning of a future landscape where personal genomic information is routinely incorporated into health care for more informed and refined therapeutic decision making.

\section{Acknowledgment}

This work was supported in part by the National Institute of General Medical Sciences (NIGMS) of the National Institutes of Health (NIH), through grant K23GM104401 (SAS), and by the National Human Genome Research Institute (NHGRI) through the eMERGE Network grant U01HG006380 (AOO, SCS, OG, SAS; Principal Investigator: EP Bottinger).

\section{Disclosure}

$\mathrm{OG}$ is a co-inventor on patent applications related to personalized clinical decision support. The other authors report no conflicts of interest in this work.

\section{References}

1. Shuldiner AR, Relling MV, Peterson JF, et al; Pharmacogenomics Research Network Translational Pharmacogenetics Program Group. The Pharmacogenomics Research Network Translational Pharmacogenetics Program: overcoming challenges of real-world implementation. Clin Pharmacol Ther. 2013;94(2):207-210.

2. Relling MV, Klein TE. CPIC: Clinical Pharmacogenetics Implementation Consortium of the Pharmacogenomics Research Network. Clin Pharmacol Ther. 2011;89(3):464-467.

3. Roberts JD, Wells GA, Le May MR, et al. Point-of-care genetic testing for personalisation of antiplatelet treatment (RAPID GENE): a prospective, randomised, proof-of-concept trial. Lancet. 2012;379(9827):1705-1711.

4. Goldspiel BR, Flegel WA, DiPatrizio G, et al. Integrating pharmacogenetic information and clinical decision support into the electronic health record. J Am Med Inform Assoc. 2014;21(3):522-528. 
5. Sanderson S, Zimmern R, Kroese M, Higgins J, Patch C, Emery J. How can the evaluation of genetic tests be enhanced? Lessons learned from the ACCE framework and evaluating genetic tests in the United Kingdom. Genet Med. 2005;7(7):495-500.

6. Koch WH. Technology platforms for pharmacogenomic diagnostic assays. Nat Rev Drug Discov. 2004;3(9):749-761.

7. King CR, Porche-Sorbet RM, Gage BF, et al. Performance of commercial platforms for rapid genotyping of polymorphisms affecting warfarin dose. Am J Clin Pathol. 2008;129(6):876-883.

8. Phillips E, Mallal S. Successful translation of pharmacogenetics into the clinic: the abacavir example. Mol Diagn Ther. 2009;13(1):1-9.

9. Burt T, Dhillon S. Pharmacogenomics in early-phase clinical development. Pharmacogenomics. 2013;14(9):1085-1097.

10. Meyer UA. Pharmacogenetics - five decades of therapeutic lessons from genetic diversity. Nat Rev Genet. 2004;5(9):669-676.

11. Dean CE. Personalized medicine: boon or budget-buster? Ann Pharmacother. 2009;43(5):958-962.

12. Welter D, MacArthur J, Morales J, et al. The NHGRI GWAS Catalog, a curated resource of SNP-trait associations. Nucleic Acids Res. 2014;42(Database issue):D1001-D1006.

13. Abrahams E, Ginsburg GS, Silver M. The Personalized Medicine Coalition: goals and strategies. Am J Pharmacogenomics. 2005;5(6): 345-355.

14. Edwards AO, Ritter R 3rd, Abel KJ, Manning A, Panhuysen C, Farrer LA. Complement factor $\mathrm{H}$ polymorphism and age-related macular degeneration. Science. 2005;308(5720):421-424.

15. Ratnapriya R, Chew EY. Age-related macular degeneration-clinical review and genetics update. Clin Genet. 2013;84(2):160-166.

16. Awh CC, Lane AM, Hawken S, Zanke B, Kim IK. CFH and ARMS2 genetic polymorphisms predict response to antioxidants and zinc in patients with age-related macular degeneration. Ophthalmology. 2013;120(11):2317-2323.

17. Singal DP, Blajchman MA. Histocompatibility (HL-A) antigens, lymphocytotoxic antibodies and tissue antibodies in patients with diabetes mellitus. Diabetes. 1973;22(6):429-432.

18. Noble JA, Valdes AM, Cook M, Klitz W, Thomson G, Erlich HA. The role of HLA class II genes in insulin-dependent diabetes mellitus: molecular analysis of 180 Caucasian, multiplex families. Am J Hum Genet. 1996;59(5):1134-1148.

19. Barrett JC, Clayton DG, Concannon P, et al; Type 1 Diabetes Genetics Consortium. Genome-wide association study and meta-analysis find that over 40 loci affect risk of type 1 diabetes. Nat Genet. 2009;41(6): 703-707.

20. Concannon P, Rich SS, Nepom GT. Genetics of type 1A diabetes. N Engl J Med. 2009;360(16):1646-1654.

21. Clayton DG. Prediction and interaction in complex disease genetics: experience in type 1 diabetes. PLoS Genet. 2009;5(7):e1000540.

22. Cho JH, Gregersen PK. Genomics and the multifactorial nature of human autoimmune disease. $N$ Engl J Med. 2011;365(17):1612-1623.

23. Manolio TA. Genomewide association studies and assessment of the risk of disease. N Engl J Med. 2010;363(2):166-176.

24. Manolio TA, Collins FS, Cox NJ, et al. Finding the missing heritability of complex diseases. Nature. 2009;461(7265):747-753.

25. van der Sijde MR, Ng A, Fu J. Systems genetics: From GWAS to disease pathways. Biochim Biophys Acta. Epub May 2, 2014.

26. D'Agostino RB Sr, Vasan RS, Pencina MJ, et al. General cardiovascular risk profile for use in primary care: the Framingham Heart Study. Circulation. 2008;117(6):743-753.

27. Easton DF, Bishop DT, Ford D, Crockford GP. Genetic linkage analysis in familial breast and ovarian cancer: results from 214 families. The Breast Cancer Linkage Consortium. Am J Hum Genet. 1993;52(4): 678-701.

28. Wooster R, Neuhausen SL, Mangion J, et al. Localization of a breast cancer susceptibility gene, BRCA2, to chromosome 13q12-13. Science. 1994;265(5181):2088-2090.

29. ACOG Committee on Practice Bulletins. Hereditary breast and ovarian cancer syndrome. Gynecol Oncol. 2009;113(1):6-11.
30. Seddon JM, Reynolds R, Maller J, Fagerness JA, Daly MJ, Rosner B. Prediction model for prevalence and incidence of advanced age-related macular degeneration based on genetic, demographic, and environmental variables. Invest Ophthalmol Vis Sci. 2009;50(5):2044-2053.

31. Dvoskin R. GPPC releases updated list of DTC genetic testing companies [press release]. Washington, DC: The Genetics and Public Policy Center; August 11, 2011. Available from: http://www.dnapolicy. org $/$ news.release.php?action=detail\&pressrelease_id=145. Accessed March 30, 2014.

32. Kalf RR, Mihaescu R, Kundu S, de Knijff P, Green RC, Janssens AC. Variations in predicted risks in personal genome testing for common complex diseases. Genet Med. 2014;16(1):85-91.

33. Ng PC, Murray SS, Levy S, Venter JC. An agenda for personalized medicine. Nature. 2009;461(7265):724-726.

34. Chua EW, Kennedy MA. Current State and Future Prospects of Directto-Consumer Pharmacogenetics. Front Pharmacol. 2012;3:152.

35. Vogel F. Moderne Problem der Humangenetik. [Modern problems of human genetics]. Ergeb Inn Med Kinderheilkd. 1959;12:52-125. German.

36. Mahgoub A, Idle JR, Dring LG, Lancaster R, Smith RL. Polymorphic hydroxylation of Debrisoquine in man. Lancet. 1977;2(8038):584-586.

37. Owen RP, Sangkuhl K, Klein TE, Altman RB. Cytochrome P450 2D6. Pharmacogenet Genomics. 2009;19(7):559-562.

38. Sim SC, Ingelman-Sundberg M. The Human Cytochrome P450 (CYP) Allele Nomenclature website: a peer-reviewed database of CYP variants and their associated effects. Hum Genomics. 2010;4(4):278-281.

39. Van Booven D, Marsh S, McLeod H, et al. Cytochrome P450 2C9CYP2C9. Pharmacogenet Genomics. 2010;20(4):277-281.

40. Scott SA, Sangkuhl K, Shuldiner AR, et al. PharmGKB summary: very important pharmacogene information for cytochrome P450, family 2, subfamily C, polypeptide 19. Pharmacogenet Genomics. 2012;22(2): $159-165$.

41. Aithal GP, Day CP, Kesteven PJ, Daly AK. Association of polymorphisms in the cytochrome P450 CYP2C9 with warfarin dose requirement and risk of bleeding complications. Lancet. 1999; 353(9154):717-719.

42. Owen RP, Gong L, Sagreiya H, Klein TE, Altman RB. VKORC1 pharmacogenomics summary. Pharmacogenet Genomics. 2010; 20(10):642-644.

43. Limdi NA, Wadelius M, Cavallari L, et al; International Warfarin Pharmacogenetics Consortium. Warfarin pharmacogenetics: a single VKORC1 polymorphism is predictive of dose across 3 racial groups. Blood. 2010;115(18):3827-3834.

44. Klein TE, Altman RB, Eriksson N, et al; International Warfarin Pharmacogenetics Consortium. Estimation of the warfarin dose with clinical and pharmacogenetic data. N Engl J Med. 2009;360(8):753-764.

45. Finkelman BS, Gage BF, Johnson JA, Brensinger CM, Kimmel SE. Genetic warfarin dosing: tables versus algorithms. J Am Coll Cardiol. 2011;57(5):612-618.

46. Pirmohamed M, Burnside G, Eriksson N, et al; EU-PACT Group. A randomized trial of genotype-guided dosing of warfarin. $N$ Engl J Med. 2013;369(24):2294-2303.

47. Kimmel SE, French B, Kasner SE, et al; COAG Investigators. A pharmacogenetic versus a clinical algorithm for warfarin dosing. $N$ Engl $J$ Med. 2013;369(24):2283-2293.

48. Zineh I, Pacanowski M, Woodcock J. Pharmacogenetics and coumarin dosing - recalibrating expectations. $N$ Engl J Med. 2013;369(24): 2273-2275.

49. Shuldiner AR, O'Connell JR, Bliden KP, et al. Association of cytochrome P450 2C19 genotype with the antiplatelet effect and clinical efficacy of clopidogrel therapy. JAMA. 2009;302(8):849-857.

50. Mega JL, Simon T, Collet JP, et al. Reduced-function CYP2C19 genotype and risk of adverse clinical outcomes among patients treated with clopidogrel predominantly for PCI: a meta-analysis. JAMA. 2010;304(16):1821-1830.

51. Lee A, Ezzeldin H, Fourie J, Diasio R. Dihydropyrimidine dehydrogenase deficiency: impact of pharmacogenetics on 5-fluorouracil therapy. Clin Adv Hematol Oncol. 2004;2(8):527-532. 
52. Wang L, Pelleymounter L, Weinshilboum R, et al. Very important pharmacogene summary: thiopurine S-methyltransferase. Pharmacogenet Genomics. 2010;20(6):401-405.

53. Barbarino JM, Haidar CE, Klein TE, Altman RB. PharmGKB summary: very important pharmacogene information for UGT1A1. Pharmacogenet Genomics. 2014;24(3):177-183.

54. Link E, Parish S, Armitage J, et al; SEARCH Collaborative Group. SLCO1B1 variants and statin-induced myopathy - a genomewide study. N Engl J Med. 2008;359(8):789-799.

55. Oshiro C, Mangravite L, Klein T, Altman R. PharmGKB very important pharmacogene: SLCO1B1. Pharmacogenet Genomics. 2010;20(3):211-216.

56. Crowley JJ, Sullivan PF, McLeod HL. Pharmacogenomic genomewide association studies: lessons learned thus far. Pharmacogenomics. 2009;10(2):161-163.

57. Motsinger-Reif AA, Jorgenson E, Relling MV, et al. Genome-wide association studies in pharmacogenomics: successes and lessons. Pharmacogenet Genomics. 2013;23(8):383-394.

58. Daly AK. Genome-wide association studies in pharmacogenomics. Nat Rev Genet. 2010;11(4):241-246.

59. Ge D, Fellay J, Thompson AJ, et al. Genetic variation in IL28B predicts hepatitis $\mathrm{C}$ treatment-induced viral clearance. Nature. 2009;461(7262):399-401.

60. Treviño LR, Shimasaki N, Yang W, et al. Germline genetic variation in an organic anion transporter polypeptide associated with methotrexate pharmacokinetics and clinical effects. J Clin Oncol. 2009;27(35):5972-5978.

61. Tantisira KG, Lasky-Su J, Harada M, et al. Genomewide association between GLCCI1 and response to glucocorticoid therapy in asthma. N Engl J Med. 2011;365(13):1173-1183.

62. Cooper GM, Johnson JA, Langaee TY, et al. A genome-wide scan for common genetic variants with a large influence on warfarin maintenance dose. Blood. 2008;112(4):1022-1027.

63. Takeuchi F, McGinnis R, Bourgeois S, et al. A genome-wide association study confirms VKORC1, CYP2C9, and CYP4F2 as principal genetic determinants of warfarin dose. PLoS Genet. 2009;5(3):e1000433.

64. Daly AK, Donaldson PT, Bhatnagar P, et al; DILIGEN Study; International SAE Consortium. HLA-B*5701 genotype is a major determinant of druginduced liver injury due to flucloxacillin. Nat Genet. 2009;41(7):816-819.

65. McCormack M, Alfirevic A, Bourgeois S, et al. HLA-A*3101 and carbamazepine-induced hypersensitivity reactions in Europeans. N Engl J Med. 2011;364(12):1134-1143.

66. Kalow W. Ethnic differences in drug metabolism. Clin Pharmacokinet. 1982;7(5):373-400.

67. Kalow W, Bertilsson L. Interethnic factors affecting drug response. Adv Drug Res. 1994;25:1-53.

68. Georgitsi M, Viennas E, Gkantouna V, et al. Population-specific documentation of pharmacogenomic markers and their allelic frequencies in FINDbase. Pharmacogenomics. 2011;12(1):49-58.

69. Scott SA. Personalizing medicine with clinical pharmacogenetics. Genet Med. 2011;13(12):987-995.

70. Evaluation of Genomic Applications in Practice and Prevention (EGAPP) Working Group. Recommendations from the EGAPP Working Group: testing for cytochrome P450 polymorphisms in adults with nonpsychotic depression treated with selective serotonin reuptake inhibitors. Genet Med. 2007;9(12):819-825.

71. Evaluation of Genomic Applications in Practice and Prevention (EGAPP) Working Group. Recommendations from the EGAPP Working Group: can UGT1A1 genotyping reduce morbidity and mortality in patients with metastatic colorectal cancer treated with irinotecan? Genet Med. 2009;11(1):15-20.

72. Swen JJ, Nijenhuis M, de Boer A, et al. Pharmacogenetics: from bench to byte - an update of guidelines. Clin Pharmacol Ther. 2011;89(5):662-673.

73. Becquemont L, Alfirevic A, Amstutz U, et al. Practical recommendations for pharmacogenomics-based prescription: 2010 ESF-UB Conference on Pharmacogenetics and Pharmacogenomics. Pharmacogenomics. 2011;12(1):113-124.
74. Teutsch SM, Bradley LA, Palomaki GE, et al; EGAPP Working Group. The Evaluation of Genomic Applications in Practice and Prevention (EGAPP) Initiative: methods of the EGAPP Working Group. Genet Med. 2009;11(1):3-14.

75. Wu AH. Genotype and phenotype concordance for pharmacogenetic tests through proficiency survey testing. Arch Pathol Lab Med. 2013;137(9):1232-1236.

76. Pratt VM, Zehnbauer B, Wilson JA, et al. Characterization of 107 genomic DNA reference materials for CYP2D6, CYP2C19, CYP2C9, VKORC1, and UGT1A1: a GeT-RM and Association for Molecular Pathology collaborative project. J Mol Diagn. 2010;12(6):835-846.

77. Dobson MG, Galvin P, Barton DE. Emerging technologies for pointof-care genetic testing. Expert Rev Mol Diagn. 2007;7(4):359-370.

78. Buchan BW, Peterson JF, Cogbill CH, et al. Evaluation of a microarraybased genotyping assay for the rapid detection of cytochrome P450 $2 \mathrm{C} 19 * 2$ and $* 3$ polymorphisms from whole blood using nanoparticle probes. Am J Clin Pathol. 2011;136(4):604-608.

79. Mallal S, Phillips E, Carosi G, et al; PREDICT-1 Study Team. HLAB*5701 screening for hypersensitivity to abacavir. $N$ Engl $J$ Med. 2008;358(6):568-579.

80. Chen P, Lin JJ, Lu CS, et al; Taiwan SJS Consortium. Carbamazepineinduced toxic effects and HLA-B*1502 screening in Taiwan. $N$ Engl $J$ Med. 2011;364(12):1126-1133.

81. Lesko LJ, Schmidt S. Clinical implementation of genetic testing in medicine: a US regulatory science perspective. Br J Clin Pharmacol. 2014;77(4):606-611.

82. Feero WG, Green ED. Genomics education for health care professionals in the 21st century. JAMA. 2011;306(9):989-990.

83. Osheroff JA, Teich JM, Middleton B, Steen EB, Wright A, Detmer DE. A roadmap for national action on clinical decision support. $J$ Am Med Inform Assoc. 2007;14(2):141-145.

84. Crews KR, Cross SJ, McCormick JN, et al. Development and implementation of a pharmacist-managed clinical pharmacogenetics service. Am J Health Syst Pharm. 2011;68(2):143-150.

85. Fernandez CA, Smith C, Yang W, et al. Concordance of DMET plus genotyping results with those of orthogonal genotyping methods. Clin Pharmacol Ther. 2012;92(3):360-365.

86. Hicks JK, Crews KR, Hoffman JM, et al. A clinician-driven automated system for integration of pharmacogenetic interpretations into an electronic medical record. Clin Pharmacol Ther. 2012;92(5):563-566.

87. Pulley JM, Denny JC, Peterson JF, et al. Operational implementation of prospective genotyping for personalized medicine: the design of the Vanderbilt PREDICT project. Clin Pharmacol Ther. 2012;92(1): $87-95$.

88. Johnson JA, Elsey AR, Clare-Salzler MJ, Nessl D, Conlon M, Nelson DR. Institutional profile: University of Florida and Shands Hospital Personalized Medicine Program: clinical implementation of pharmacogenetics. Pharmacogenomics. 2013;14(7):723-726.

89. Johnson JA, Burkley BM, Langaee TY, Clare-Salzler MJ, Klein TE, Altman RB. Implementing personalized medicine: development of a cost-effective customized pharmacogenetics genotyping array. Clin Pharmacol Ther. 2012;92(4):437-439.

90. O'Donnell PH, Bush A, Spitz J, et al. The 1200 patients project: creating a new medical model system for clinical implementation of pharmacogenomics. Clin Pharmacol Ther. 2012;92(4):446-449.

91. Ratain MJ. Personalized medicine: building the GPS to take us there. Clin Pharmacol Ther. 2007;81(3):321-322.

92. Gottesman O, Scott SA, Ellis SB, et al. The CLIPMERGE PGx Program: clinical implementation of personalized medicine through electronic health records and genomics-pharmacogenomics. Clin Pharmacol Ther. 2013;94(2):214-217.

93. Overby CL, Erwin AL, Abul-Husn NS, et al. Physician Attitudes toward Adopting Genome-Guided Prescribing through Clinical Decision Support. J Pers Med. 2014;4(1):35-49.

94. Keller MA, Gordon ES, Stack CB, et al. Coriell Personalized Medicine Collaborative $^{\circledR}$ : a prospective study of the utility of personalized medicine. Per Med. 2010;7(3):301-317. 
95. Stack CB, Gharani N, Gordon ES, Schmidlen T, Christman MF, Keller MA. Genetic risk estimation in the Coriell Personalized Medicine Collaborative. Genet Med. 2011;13(2):131-139.

96. Gharani N, Keller MA, Stack CB, et al. The Coriell personalized medicine collaborative pharmacogenomics appraisal, evidence scoring and interpretation system. Genome Med. 2013;5(10):93.

97. Lerman C, Croyle RT, Tercyak KP, Hamann H. Genetic testing: psychological aspects and implications. J Consult Clin Psychol. 2002;70(3):784-797.

98. Ward RL, Hicks S, Hawkins NJ. Population-based molecular screening for Lynch syndrome: implications for personalized medicine. J Clin Oncol. 2013;31(20):2554-2562.

99. Meiser B. Psychological impact of genetic testing for cancer susceptibility: an update of the literature. Psychooncology. 2005; 14(12):1060-1074.

100. Creighton S, Almqvist EW, MacGregor D, et al. Predictive, pre-natal and diagnostic genetic testing for Huntington's disease: the experience in Canada from 1987 to 2000. Clin Genet. 2003;63(6):462-475.

101. Morrison PJ, Harding-Lester S, Bradley A. Uptake of Huntington disease predictive testing in a complete population. Clin Genet. 2011; 80(3):281-286.

102. Roberts JS, Barber M, Brown TM, et al. Who seeks genetic susceptibility testing for Alzheimer's disease? Findings from a multisite, randomized clinical trial. Genet Med. 2004;6(4):197-203.

103. Foster MW, Mulvihill JJ, Sharp RR. Evaluating the utility of personal genomic information. Genet Med. 2009;11(8):570-574.

104. Roberts JS, Ostergren J. Direct-to-Consumer Genetic Testing and Personal Genomics Services: A Review of Recent Empirical Studies. Curr Genet Med Rep. 2013;1(3):182-200.

105. Bloss CS, Schork NJ, Topol EJ. Effect of direct-to-consumer genomewide profiling to assess disease risk. $N$ Engl J Med. 2011;364(6): 524-534.

106. Giovanni MA, Fickie MR, Lehmann LS, et al. Health-care referrals from direct-to-consumer genetic testing. Genet Test Mol Biomarkers. 2010;14(6):817-819.

107. Kaufman DJ, Bollinger JM, Dvoskin RL, Scott JA. Risky business: risk perception and the use of medical services among customers of DTC personal genetic testing. J Genet Couns. 2012;21(3):413-422.

108. Haga SB, O'Daniel JM, Tindall GM, Lipkus IR, Agans R. Survey of US public attitudes toward pharmacogenetic testing. Pharmacogenomics J. 2012;12(3):197-204.

109. Sanderson SC, Wardle J. Associations between anticipated reactions to genetic test results and interest in genetic testing: will self-selection reduce the potential for harm? Genet Test. 2008;12(1):59-66.

110. Haga SB, Tindall G, O’Daniel JM. Public perspectives about pharmacogenetic testing and managing ancillary findings. Genet Test Mol Biomarkers. 2012;16(3):193-197.

111. Sanderson SC, Humphries SE, Hubbart C, Hughes E, Jarvis MJ, Wardle J. Psychological and behavioural impact of genetic testing smokers for lung cancer risk: a phase II exploratory trial. $J$ Health Psychol. 2008;13(4):481-494.

112. Marteau TM, French DP, Griffin SJ, et al. Effects of communicating DNA-based disease risk estimates on risk-reducing behaviours [review]. Cochrane Database Syst Rev. 2010;10:CD007275.

113. Vernarelli JA, Roberts JS, Hiraki S, Chen CA, Cupples LA, Green RC. Effect of Alzheimer disease genetic risk disclosure on dietary supplement use. Am J Clin Nutr. 2010;91(5):1402-1407.

114. Zick CD, Mathews CJ, Roberts JS, Cook-Deegan R, Pokorski RJ, Green RC. Genetic testing for Alzheimer's disease and its impact on insurance purchasing behavior. Health Aff (Millwood). 2005;24(2): 483-490.

115. Haga SB, LaPointe NM. The potential impact of pharmacogenetic testing on medication adherence. Pharmacogenomics J. 2013;13(6): 481-483.

116. Schwartz SM, Schwartz HT, Horvath S, Schadt E, Lee SI. A systematic approach to multifactorial cardiovascular disease: causal analysis. Arterioscler Thromb Vasc Biol. 2012;32(12):2821-2835.
117. Hayes DF, Markus HS, Leslie RD, Topol EJ. Personalized medicine: risk prediction, targeted therapies and mobile health technology. $B M C$ Med. 2014; 12:37.

118. Clancy JP, Johnson SG, Yee SW, et al. Clinical Pharmacogenetics Implementation Consortium (CPIC) guidelines for ivacaftor therapy in the context of CFTR genotype. Clin Pharmacol Ther. 2014;95(6): 592-597.

119. Flockhart DA, O'Kane D, Williams MS, et al; ACMG Working Group on Pharmacogenetic Testing of CYP2C9, VKORC1 Alleles for Warfarin Use. Pharmacogenetic testing of CYP2C9 and VKORC1 alleles for warfarin. Genet Med. 2008;10(2):139-150.

120. Johnson JA, Gong L, Whirl-Carrillo M, et al; Clinical Pharmacogenetics Implementation Consortium. Clinical Pharmacogenetics Implementation Consortium Guidelines for CYP2C9 and VKORC1 genotypes and warfarin dosing. Clin Pharmacol Ther. 2011;90(4):625-629.

121. Scott SA, Sangkuhl K, Gardner EE, et al; Clinical Pharmacogenetics Implementation Consortium. Clinical Pharmacogenetics Implementation Consortium guidelines for cytochrome P450-2C19 (CYP2C19) genotype and clopidogrel therapy. Clin Pharmacol Ther. 2011;90(2):328-332.

122. Scott SA, Sangkuhl K, Stein CM, et al; Clinical Pharmacogenetics Implementation Consortium. Clinical Pharmacogenetics Implementation Consortium guidelines for CYP2C19 genotype and clopidogrel therapy: 2013 update. Clin Pharmacol Ther. 2013;94(3):317-323.

123. Holmes DR, Dehmer GJ, Kaul S, Leifer D, O'Gara PT, Stein CM. ACCF/AHA clopidogrel clinical alert: approaches to the FDA "boxed warning": a report of the American College of Cardiology Foundation Task Force on clinical expert consensus documents and the American Heart Association endorsed by the Society for Cardiovascular Angiography and Interventions and the Society of Thoracic Surgeons. $J$ Am Coll Cardiol. 2010;56(4):321-341.

124. Hicks JK, Swen JJ, Thorn CF, et al; Clinical Pharmacogenetics Implementation Consortium. Clinical Pharmacogenetics Implementation Consortium guideline for CYP2D6 and CYP2C19 genotypes and dosing of tricyclic antidepressants. Clin Pharmacol Ther. 2013;93(5):402-408.

125. Crews KR, Gaedigk A, Dunnenberger HM, et al; Clinical Pharmacogenetics Implementation Consortium. Clinical Pharmacogenetics Implementation Consortium (CPIC) guidelines for codeine therapy in the context of cytochrome P450 2D6 (CYP2D6) genotype. Clin Pharmacol Ther. 2012;91(2):321-326.

126. Lyon E, Gastier Foster J, Palomaki GE, et al; working group of the Molecular Genetics Subcommittee on behalf of the American College of Medical Genetics and Genomics ACMG) Laboratory Quality Assurance Committee. Laboratory testing of CYP2D6 alleles in relation to tamoxifen therapy. Genet Med. 2012;14(12):990-1000.

127. Caudle KE, Thorn CF, Klein TE, et al. Clinical Pharmacogenetics Implementation Consortium guidelines for dihydropyrimidine dehydrogenase genotype and fluoropyrimidine dosing. Clin Pharmacol Ther. 2013;94(6):640-645.

128. Martin MA, Klein TE, Dong BJ, Pirmohamed M, Haas DW, Kroetz DL; Clinical Pharmacogenetics Implementation Consortium. Clinical pharmacogenetics implementation consortium guidelines for HLA-B genotype and abacavir dosing. Clin Pharmacol Ther. 2012;91(4):734-738.

129. Hershfield MS, Callaghan JT, Tassaneeyakul W, et al. Clinical Pharmacogenetics Implementation Consortium guidelines for human leukocyte antigen-B genotype and allopurinol dosing. Clin Pharmacol Ther. 2013;93(2):153-158.

130. Leckband SG, Kelsoe JR, Dunnenberger HM, et al; Clinical Pharmacogenetics Implementation Consortium. Clinical Pharmacogenetics Implementation Consortium guidelines for HLA-B genotype and carbamazepine dosing. Clin Pharmacol Ther. 2013;94(3):324-328.

131. Muir AJ, Gong L, Johnson SG, et al; Clinical Pharmacogenetics Implementation Consortium (CPIC). Clinical Pharmacogenetics Implementation Consortium (CPIC) guidelines for IFNL3 (IL28B) genotype and PEG interferon- $\alpha$-based regimens. Clin Pharmacol Ther. 2014;95(2):141-146. 
132. Wilke RA, Ramsey LB, Johnson SG, et al; Clinical Pharmacogenomics Implementation Consortium (CPIC). The clinical pharmacogenomics implementation consortium: CPIC guideline for SLCO1B1 and simvastatin-induced myopathy. Clin Pharmacol Ther. 2012;92(1):112-117.

133. Relling MV, Gardner EE, Sandborn WJ, et al; Clinical Pharmacogenetics Implementation Consortium. Clinical Pharmacogenetics Implementation Consortium guidelines for thiopurine methyltransferase genotype and thiopurine dosing. Clin Pharmacol Ther. 2011;89(3):387-391.
134. Relling MV, Gardner EE, Sandborn WJ, et al. Clinical pharmacogenetics implementation consortium guidelines for thiopurine methyltransferase genotype and thiopurine dosing: 2013 update. Clin Pharmacol Ther. 2013;93(4):324-325.

135. Raimundo $\mathrm{S}$, Toscano $\mathrm{C}$, Klein $\mathrm{K}$, et al. A novel intronic mutation, $2988 \mathrm{G}>\mathrm{A}$, with high predictivity for impaired function of cytochrome P450 2D6 in white subjects. Clin Pharmacol Ther. 2004;76(2): $128-138$.

\section{Publish your work in this journal}

Pharmacogenomics and Personalized Medicine is an international, peerreviewed, open access journal characterizing the influence of genotype on pharmacology leading to the development of personalized treatment programs and individualized drug selection for improved safety, efficacy and sustainability. This journal is indexed on the American Chemical
Society's Chemical Abstracts Service (CAS). The manuscript management system is completely online and includes a very quick and fair peer-review system, which is all easy to use. Visit http://www.dovepress. com/testimonials.php to read real quotes from published authors.

Submit your manuscript here: http://www.dovepress.com/pharmacogenomics-and-personalized-medicine-journal 